\title{
IgG4-Related Airway Involvement Which Developed in a Patient Receiving Corticosteroid Therapy for Autoimmune Pancreatitis
}

\author{
Hiroshi Yamamoto ${ }^{1}$, Masanori Yasuo ${ }^{1}$, Yayoi Nomura ${ }^{1}$, Toshihiko Agatsuma ${ }^{1}$, \\ Atsuhito Ushiki ${ }^{1}$, Toshiki Yokoyama ${ }^{1}$, Kazuhisa Urushihata ${ }^{1}$, Masayuki Hanaoka ${ }^{1}$, \\ Tomonobu Koizumi ${ }^{1}$, Mai Iwaya ${ }^{2}$, Akihiko Yoshizawa ${ }^{2}$, Satoshi Kawakami ${ }^{3}$, \\ Hideaki Hamano $^{4}$, Shigeyuki Kawa ${ }^{5}$ and Keishi Kubo ${ }^{1}$
}

\begin{abstract}
A 66-year-old man was diagnosed with autoimmune pancreatitis in February 2009 and started $40 \mathrm{mg}$ of oral prednisolone followed by a maintenance dose of $5 \mathrm{mg}$ daily. The patient developed a cough in October 2010 and visited our division. He had a high serum concentration of immunoglobulin (Ig) G4 and his chest computed tomography showed airway stenosis without bilateral hilar lymphadenopathy (BHL). The bronchial biopsy specimens revealed lymphoplasmacytic infiltrations with IgG4-positive/IgG-positive plasma cells of more than 50\%. Thus, we diagnosed the airway lesion with IgG4-related airway involvement. This is the first report of a patient with IgG4-related airway involvement without BHL.
\end{abstract}

Key words: airway involvement, bilateral hilar lymphadenopathy (BHL), lymphoplasmacytic, IgG4, autoimmune pancreatitis (AIP)

(Intern Med 50: 3023-3026, 2011)

(DOI: 10.2169/internalmedicine.50.6220)

\section{Introduction}

Immunoglobulin (Ig) G4-related lung disease has attracted increasing attention (1). Autoimmune pancreatitis (AIP) is characterized by a high serum IgG4 concentration and various types of extrapancreatic involvement including the lung $(2,3)$. Therefore, it might be considered that AIP is not simply pancreatitis but that AIP is a pancreatic lesion involved in IgG4-related disease with systemic organ involvement $(4,5)$. In 2009, we reported the first patient with airway involvement in IgG4-related AIP showing central airway stenosis and bilateral hilar lymphadenopathy (BHL) similar to sarcoidosis (6).

We herein report a patient who was already diagnosed with AIP and had been treated by oral prednisolone with a maintenance dose of $5 \mathrm{mg}$ developed cough. The patient showed bronchial wall thickening without BHL on computed tomography (CT) which was identified to be IgG4related airway involvement.

\section{Case Report}

A 66-year-old man was admitted to our respiratory division (Shinshu University Hospital, Matsumoto, Japan) complaining of cough without fever and dyspnea in December 2010. He had no family history of pancreatic disease, collagen disease, or autoimmune disease. He had a 45 pack-year history of smoking and a history of submandibular gland swelling when he was in his 50's. In February 2009, he was

\footnotetext{
${ }^{1}$ The First Department of Internal Medicine, Shinshu University School of Medicine, Japan, ${ }^{2}$ Department of Laboratory Medicine, Shinshu University School of Medicine, Japan, ${ }^{3}$ Department of Radiology, Shinshu University School of Medicine, Japan, ${ }^{4}$ Department of Internal Medicine, Gastroenterology, Shinshu University School of Medicine, Japan and ${ }^{5}$ Center for Health, Safety and Environmental Management, Shinshu University School of Medicine, Japan

Received for publication July 20, 2011; Accepted for publication September 11, 2011

Correspondence to Dr. Hiroshi Yamamoto, yama5252@hsp.md.shinshu-u.ac.jp
} 

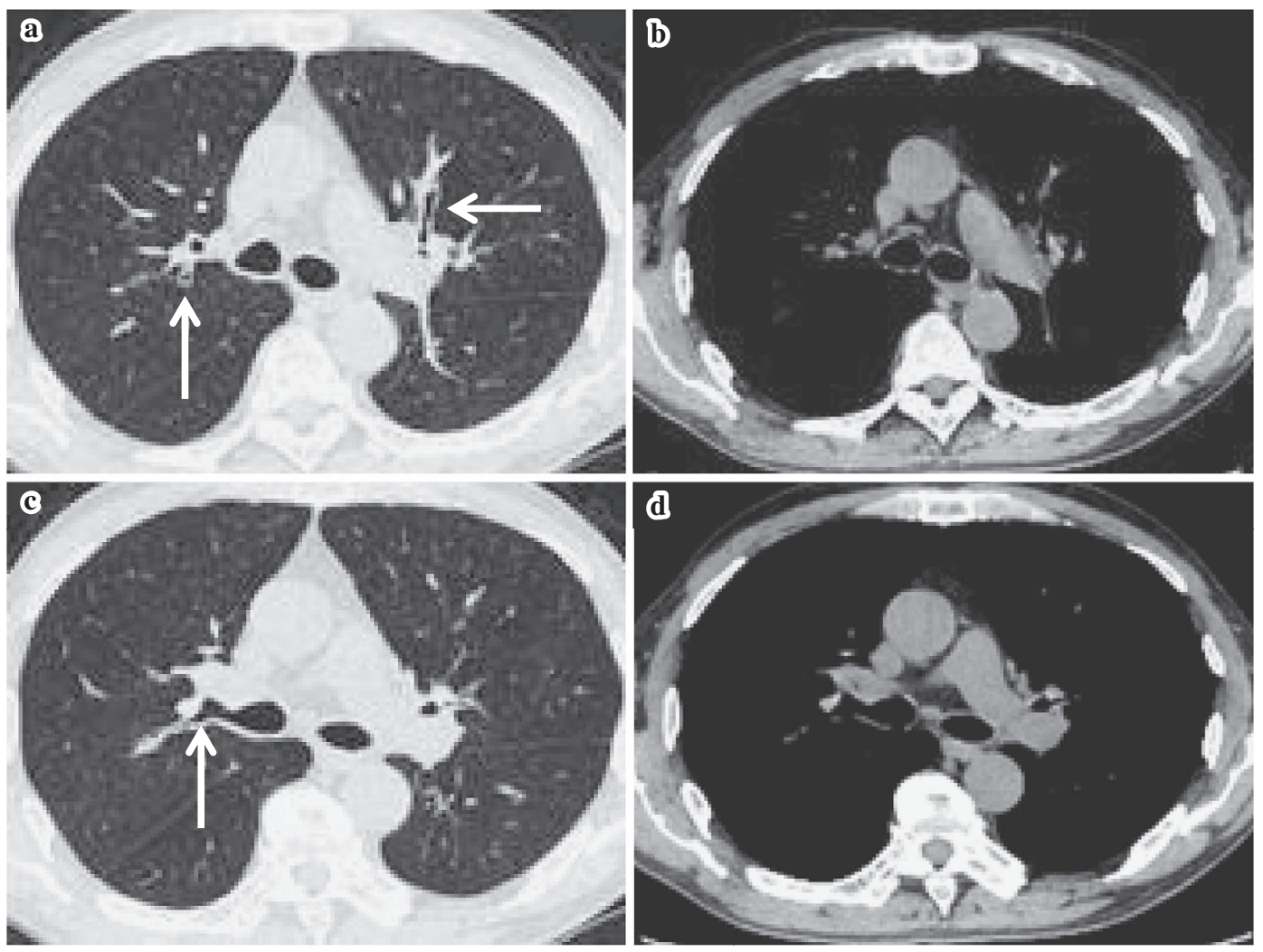

Figure 1. Chest CT scanning showed bronchial wall thickening (a, c) without swelling of hilar or mediastinal lymph nodes (b, d). Arrows indicate the thickened airways.

diagnosed as autoimmune pancreatitis (AIP) without lung manifestation based on the criteria of AIP proposed by Japanese Pancreatic Society in 2006 (2). Thus, he received 40 $\mathrm{mg}$ of oral prednisolone for 4 weeks from that time, followed by a reduction in dose by $5 \mathrm{mg}$ per week over 7 weeks until a daily maintenance dose of $5 \mathrm{mg}$ was reached; this was the dose at this admission. He had no history of stridor and no wheezing was heard in his chest.

Laboratory examinations showed the following values (normal range): peripheral white blood cell count, $2,430 / \mu \mathrm{L}$; C-reactive protein, $0.02 \mathrm{mg} / \mathrm{dL}$; total protein (TP), $6.9 \mathrm{~g} / \mathrm{dL}$; albumin, $4.1 \mathrm{~g} / \mathrm{dL}$; lactate dehydrogenase (LD), $206 \mathrm{IU} / \mathrm{L}$ $(<220)$; IgE, $25 \mathrm{IU} / \mathrm{L}(<250)$; and $\mathrm{IgG}, 1,585 \mathrm{mg} / \mathrm{dL}(<$ 1,700). The serum concentrations of the IgG subclasses were as follows: IgG1, $799 \mathrm{mg} / \mathrm{dL}(<1,080)$; IgG2, $873 \mathrm{mg} /$ dL (<931); IgG3, $34 \mathrm{mg} / \mathrm{dL}(<121)$; and IgG4, $513 \mathrm{mg} / \mathrm{dL}$ $(<108)$, and IgG4/IgG $32.3 \%$. The serum autoantibodies were all negative, including antinuclear antibody (ANA), rheumatoid factor (RF), anti-double-stranded DNA antibody, anti-RNP antibody, anti-Sjögren's syndrome (SS)-A antibody, SS-B antibody, and anti-neutrophil cytoplasmic autoantibody (ANCA). The serum soluble interleukin-2 receptor was $438 \mathrm{U} / \mathrm{mL}(<421)$ and the serum angiotensinconverting enzyme (ACE) was 8.6 U/L $(<25)$. His electrophoretogram of a blood sample did not show any abnormal bands. His thyroid function was normal and the level of brain natriuretic peptide (BNP) in the blood was normal. Proteinuria was not observed. A pulmonary function test (PFT) revealed a vital capacity of $4.06 \mathrm{~L}(112.8 \%$ pre- dicted), forced expiratory volume in one second $\left(\mathrm{FEV}_{1}\right)$ of $2.89 \mathrm{~L}$ (100.3\% pred.), $\mathrm{FEV}_{1} /$ forced vital capacity (FVC) of $69.0 \%$, peak expiratory flow (PEF) of $5.80 \mathrm{~L} / \mathrm{sec}(73.1 \%$ pred.), and a diffusing capacity of the lung for carbon monoxide of $18.14 \mathrm{~mL} / \mathrm{min} / \mathrm{mmHg}(74.1 \%$ pred.) without significant reversibility (7). After inhalation of bronchodilator, PFT revealed $\mathrm{FEV}_{1}$ of $3.18 \mathrm{~L}\left(110.4 \%\right.$ pred.) and $\mathrm{FEV}_{1} /$ forced vital capacity (FVC) of $77.8 \%$.

A chest X-ray showed tram lines in bilateral lungs without infiltration and pleural effusion. The chest CT showed bronchial wall thickening without hilar lymph node swelling (Fig. 1). In order to diagnose the airway lesion, fiberoptic bronchoscopy (BF 1T-260, Olympus Corporation, Tokyo, Japan) was performed and bronchoalveolar lavage (BAL) and bronchial biopsy at the right second bifurcation were achieved according to our routine protocol (8). Bronchoscopic examination unveiled a mild airway stenosis accompanied with mucosal edema from main bronchus to the upper and lower lobe bronchus on both sides of the lung (Fig. 2). Analysis of the bronchoalveolar lavage fluid revealed a cell count of $2.3 \times 10^{4} / \mathrm{mL}$, with $72.0 \%$ macrophages, $24.5 \%$ lymphocytes (CD4/8 ratio 1.14), $1.7 \%$ eosinophils, and $0.9 \%$ neutrophils. The bronchial biopsy specimens of the patient revealed diffuse airway inflammatory infiltrates, consisting mainly of plasma cells and lymphocytes without typical granuloma. There were scattered eosinophils in the bronchial tissues of the patient; typical findings of asthma, such as goblet cell hyperplasia and subepithelial fibrosis (9), were not observed in any of the 
bronchial biopsy specimens (Fig. 3a). The immunohistochemical examinations showed diffuse infiltration of IgG4positive plasma cells in the specimens (Fig. 3b). The numbers of both IgG4-positive and IgG-positive cells were counted in regions of the highest cellular density and averaged for three high-power fields (HPF) by one pathologist (A. Y.) (3). The number of IgG4-positive plasma cells per $\mathrm{HPF}$ scored as moderate (10-30/HPF) according to $\mathrm{Ka}-$ misawa et al (4), and the percentage of $\mathrm{IgG} 4$-positive to IgG-positive plasma cells (IgG4+/IgG+) was 68.5\%. Therefore, the airway lesion of this patient was diagnosed as $\mathrm{IgG}$ 4-related airway involvement. He has been treated with salmeterol/fluticasone combination (SFC: salmeterol $100 \mu \mathrm{g} /$ day and fluticasone $500 \mu \mathrm{g} /$ day) inhalation in addition to 5 $\mathrm{mg}$ of oral prednisolone. As the result of the therapy, his cough has disappeared. However, several months after the occurrence of IgG4-related airway involvement, this patient had redeveloped the AIP with serum IgG4 elevation. Thus, he received $40 \mathrm{mg}$ of oral prednisolone, again.



Figure 2. Bronchoscopic examination revealed the irregular stenosis with mucosal edema in the right second bifurcation of the bronchus.

Discussion

We herein described that an elderly male diagnosed as AIP treated by oral corticosteroid at maintenance dose who developed a cough.

Although rigorous diagnostic criteria for IgG4-related lung disease have not been established, a high serum concentration of IgG4 and marked IgG4-positive plasma cell infiltration in the biopsy specimens are accepted to be the characteristic features of this disease (1). It was reported that the lung lesions were detected in 25 (54\%) out of 46 patients with AIP who had undergone thin-slice chest CT scanning (10). Furthermore, BHL was revealed in 54 (78.3\%) of 69 patients with AIP undergoing thoracic CT and the lymphadenopathy disappeared in all cases after corticosteroid therapy (10). Although they did not approach the BHL of the 54 patients with AIP, it was reported that the biopsy specimens of BHL in patients with AIP, taken by the endoscopic ultrasound-guided fine needle aspiration (EUSFNA) technique, revealed IgG4-bearing plasma cell infiltration without epithelioid cell granuloma (11). We think that the BHL of a patient with IgG4-related airway involvement represents one of the manifestations of IgG4-related disease. Masaki et al reported that $6(9.4 \%)$ of 64 patients with Mikulicz's disease showed interstitial lung disease and 11 (17.2\%) of 64 patients had AIP (5). Furthermore, they proposed a new clinical entity, IgG4-positive multiorgan lymphoproliferative syndrome (IgG4+MOLPS), a syndrome characterized by hyper-IgG4 gammaglobulinemia (>135 mg/ $\mathrm{dL})$ and $\mathrm{IgG} 4-$ positive plasma cell infiltration in the tissues (IgG4+/IgG+ $>50 \%$ ) (5). On the other hand, Zen et al reported 21 patients with IgG4-related lung and pleural disease diagnosed on the basis of the histopathological findings of the lung and pleura (1). Radiologically, they categorized IgG4-related lung disease into 4 major subtypes: solid nodular type having a solitary nodular lesion that included a mass (similar to lung cancer); round-shaped ground-glass
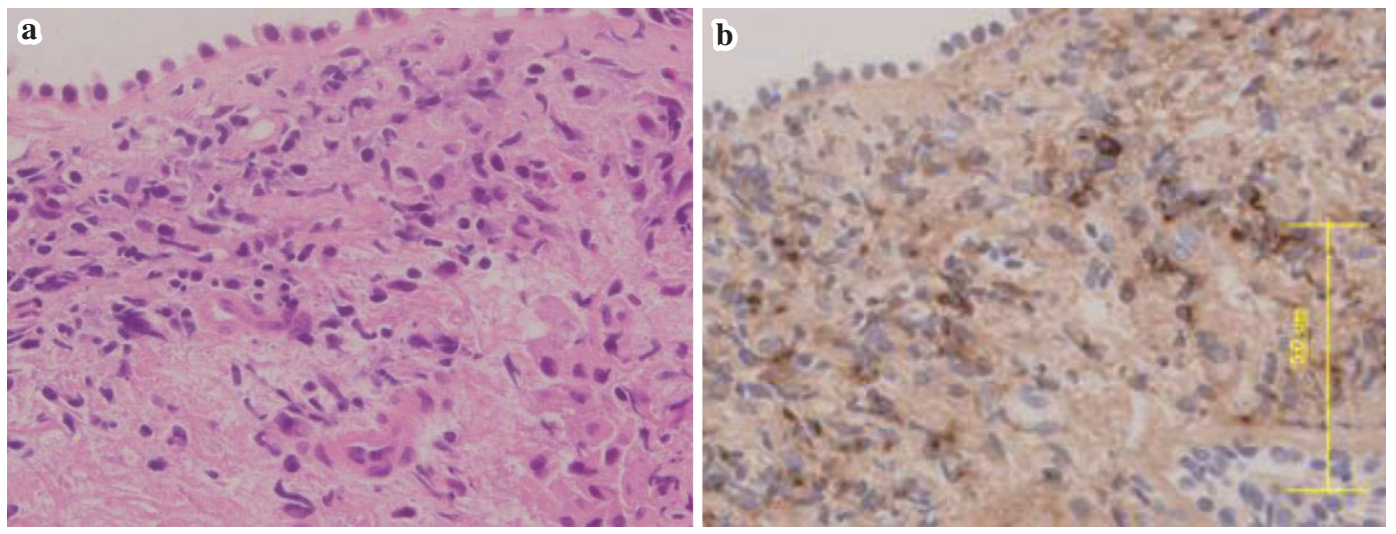

Figure 3. Hematoxylin and Eosin (HE) staining showed diffuse airway inflammatory infiltrates, consisting of plasma cells, lymphocytes, and scattered eosinophils. No typical granuloma or mucousgland hyperplasia were found in the bronchial biopsy specimen (a). Immunohistochemical staining showed the marked infiltration of IgG4-positive plasma cells (b) in the specimens. Bar $=50 \mu \mathrm{m}$ 
opacity (GGO) type characterized by multiple round-shaped GGOs (similar to bronchiolo-alveolar carcinoma); alveolar interstitial type showing bronchiectasis and diffuse GGO (similar to non-specific interstitial pneumonia); and bronchovascular type showing thickening of bronchovascular bundles and interlobular septa (similar to sarcoidosis) (1). It is important to distinguish IgG4-related lung disease from the other diseases. Pathologically, the lung and pleural lesions of the 21 patients had characteristic HE findings such as diffuse lymphoplasmacytic infiltration, irregular fibrosis, and scattered eosinophil infiltration. The immunostaining showed diffuse IgG4-positive plasma cell infiltration and a ratio of IgG4+/IgG+ greater than $30 \%$ (1). The biopsy specimens of the bronchus in the present patient showed diffuse IgG4positive plasma cell infiltrations, in agreement with the criteria for IgG4-related lung diseases proposed by Zen et al (1) and IgG4+MOLPS (5). Thus, we diagnosed the bronchial manifestation in this patient as IgG4-related airway involvement. We think that the airway lesion of this patient is not typical bronchial asthma because he did not have stridor, wheezing, significant reversibility in PET, and typical pathological findings of asthma in his bronchial mucosa.

It was reported that lung involvement can occur not only "after" and "at the same time" but also "before" pancreatic involvement of patients with IgG4-related AIP (3). In the present patient, the lung manifestation was not detected at the onset of AIP. However, the recurrence of pancreatic manifestation was not observed at the occurrence of IgG4related airway involvement. Interestingly, his AIP had reactivated with serum IgG4 elevation following the development of IgG4-related airway involvement by several months.

Recently, we described the clinical features of 6 patients with central airway involvement in AIP (12). Because the 6 patients met the criteria of IgG4-related lung disease proposed by Zen et al (1), we think that the central airway involvement in AIP is an IgG4-related airway lesion belonging to IgG4-related lung diseases. The chest CT of all 6 patients showed bronchial wall thickening and significant BHL. It was interesting that the present patient with an $\mathrm{IgG}$ 4-related airway lesion did not show BHL. It seems that various lesions, such as airway involvement and hilar lymph node lesion, have not only asynchronous development but also different therapeutic responses to corticosteroid therapy.
To the best of our knowledge, this is the first report of a patient with IgG4-related airway involvement without BHL. We should not forget that IgG4-related airway involvement is one of the important differential diagnoses of cough in a patient who has history of IgG4-related disease, even when the patient receives corticosteroid therapy without the recurrence of extra airway manifestations.

The authors state that they have no Conflict of Interest (COI).

\section{References}

1. Zen Y, Inoue D, Kitao A, et al. IgG4-related lung and pleural disease: a clinicopathologic study of 21 cases. Am J Surg Pathol 33: 1886-1893, 2009.

2. Kawa S, Hamano H. Clinical features of autoimmune pancreatitis. J Gastroenterol 42 (Suppl 18): 9-14, 2007.

3. Shrestha B, Sekiguchi H, Colby TV, et al. Distinctive pulmonary histopathology with increased IgG4-positive plasma cells in patients with autoimmune pancreatitis: report of 6 and 12 cases with similar histopathology. Am J Surg Pathol 33: 1450-1462, 2009.

4. Kamisawa T, Funata N, Hayashi Y, et al. A new clinicopathological entity of IgG4-related autoimmune disease. J Gastroenterol 38: 982-984, 2003.

5. Masaki Y, Dong L, Kurose N, et al. Proposal for a new clinical entity, IgG4-positive multiorgan lymphoproliferative syndrome: analysis of 64 cases of IgG4-related disorders. Ann Rheum Dis 68: 1310-1315, 2009.

6. Ito $\mathrm{M}$, Yasuo $\mathrm{M}$, Yamamoto $\mathrm{H}$, et al. Central airway stenosis in a patient with autoimmune pancreatitis. Eur Respir J 33: 680-683, 2009.

7. American Thoracic Society. Lung function testing: selection of reference values and interpretative strategies. Am Rev Respir Dis 144: 1202-1218, 1991.

8. Tsushima $\mathrm{K}$, Tanabe $\mathrm{T}$, Yamamoto $\mathrm{H}$, et al. Pulmonary involvement of autoimmune pancreatitis. Eur J Clin Invest 39: 714-722, 2009.

9. Siddiqui S, Martin JG. Structural aspects of airway remodeling in asthma. Curr Allergy Asthma Rep 8: 540-547, 2008.

10. Fujinaga $Y$, Kadoya M, Kawa $S$, et al. Characteristic findings in images of extra-pancreatic lesions associated with autoimmune pancreatitis. Eur J Radiol 76: 228-238, 2010.

11. Ando N, Yasuda I, Saito M, Moriwaki H. Hilar lymphadenopathy associated with autoimmune pancreatitis. Pancreas 33: 101-102, 2006.

12. Yamamoto $H$, Yasuo $M$, Ito $M$, et al. Clinical features of central airway involvement in autoimmune pancreatitis. Eur Respir J 38: 1233-1236, 2011.

(C) 2011 The Japanese Society of Internal Medicine http://www.naika.or.jp/imindex.html 